BULLETIN (New Series) OF THE

AMERICAN MATHEMATICAL SOCIETY

Volume 40, Number 2, Pages 203-228

S 0273-0979(03)00981-9

Article electronically published on February 12, 2003

\title{
LOGICAL DREAMS
}

\author{
SAHARON SHELAH
}

\begin{abstract}
We discuss the past and future of set theory, axiom systems and
\end{abstract} independence results. We deal in particular with cardinal arithmetic.

Reading instructions: The real instructions, i.e., my hopes, are that you start at the beginning and read until the end. But if you are looking for advice on how much not to read and still get what you like, note that the sections are essentially independent. If you wonder what the relevance of mathematical logic is to the rest of mathematics and whether you implicitly accept the usual axioms of set theory (that is, the axioms of E. Zermelo and A. Fraenkel with the Axiom of Choice, from now on referred to as ZFC), you should read mainly $\S 1$.

If you are excited about the independence phenomenon, you should read $\S 2$. If you are interested in considerations on new additional axioms and/or in looking at definable sets of reals (as in descriptive set theory) as compared to general sets of reals, and in looking more deeply into independence, you should go to $\S 3$. For more on independence in set theory, see $\S 4$. If you really are interested in cardinal arithmetic, i.e., the arithmetic of infinite numbers of G. Cantor, go to $\S 5$.

I had intended to also write sections on "Pure model theory", "Applied model theory", and "On Speculations and Nonsense", but only $\S 6$ materialized.

I thank J. Baldwin, A. Blass, G. Cherlin, P. Eklof, U. Hrushovski, J. Kennedy, C. Laskowski, A. Levy, A. Rosłanowski, J. Väänänen and A. Villaveces for many comments.

\section{$\S 0$ INTRODUCTORY REMARKS}

The intention to set the agenda for the 21st century for mathematical logic is certainly overly ambitious, if not to say megalomaniac. Unavoidably, I will speak mostly on directions I am interested in and/or relatively knowledgeable about (which are quite close to those I have worked on), so this selection will be riddled with prejudices, but at least they are mine; hopefully some others will be infuriated enough to offer differing opinions.

I will try to make this article accessible to any mathematician (if (s)he ignores some more specialized parts), as this is intended for a general audience (of mathematicians); this differs from my recent papers on open problems, Sh 666], Sh 702].

2000 Mathematics Subject Classification. Primary 03-02, 03Bxx, 03Exx; Secondary 03Cxx, $03 \mathrm{C} 45$.

Key words and phrases. Mathematical logic, set theory, independence, incompleteness, forcing, large cardinals.

I would like to thank Alice Leonhardt for the beautiful typing. This paper is based on my lecture (and the preparations to the lecture) during the conference Mathematical Challenges of the 21st Century and is publication E23.

(C)2003 American Mathematical Society 
Also, as readers tend to get lost, each section makes a fresh start and even subsections can be read separately (so from a negative point of view, there are repetitions and a somewhat fragmented character).

Usually we try to appeal to non-expert readers, do our best, fail, and barely make it interesting for the professional, but I am doomed to make the attempt. Many of the issues discussed below have been very popular and everyone has an opinion; I will mention some other opinions in order to disagree with them.

Cardinal Arithmetic: We shall deal several times with cardinal arithmetic, so recall that two (possibly infinite) sets $A, B$ are called equinumerous (in short: $A \sim B$ ) if there is a one-to-one mapping from one onto the other. Essentially, a cardinal number $\lambda$ is $A / \sim$ written as $|A|$. We define addition, multiplication and exponentiation of those numbers by

$$
\begin{aligned}
& |A|+|B|=|A \cup B|, \text { when } A, B \text { are disjoint; } \\
& |A| \times|B|=|A \times B| ; \\
& |A|^{|B|}=\left|{ }^{B} A\right|=\mid\{f: f \text { a function from } B \text { into } A\} \mid ; \text { also } \\
& \prod_{i \in I}\left|A_{i}\right|=\left|\prod_{i \in I} A_{i}\right| .
\end{aligned}
$$

Then all the usual equalities and weak $(\leq)$ inequalities hold, but for infinite cardinals $\lambda, \mu$ we have $\lambda+1=\lambda, \lambda+\mu=\max \{\lambda, \mu\}=\lambda \times \mu$, but $2^{\lambda}>\lambda$ for every $\lambda$.

Let $\aleph_{0}$ be the number of natural numbers, $\aleph_{n+1}$ the successor of $\aleph_{n}, \aleph_{\omega}=$ $\sum\left\{\aleph_{n}: n \in \mathbb{N}\right\}$. The Continuum Hypothesis $(\mathrm{CH})$ is the statement $2^{\aleph_{0}}=\aleph_{1}$, and the Generalized Continuum Hypothesis $\left(\mathrm{GCH}\right.$ ) says that $2^{\lambda}$ is $\lambda^{+}$(the successor of $\lambda$ ) for every infinite $\lambda$. In fact, GCH says that not only are the rules of addition and multiplication of infinite numbers simple but also the rules of exponentiation.

An ordinal is the order type of a linear order which is well ordered, i.e., whose every non-empty subset has a first element. In fact we can choose a representative; for ordinals $\alpha, \beta$ the meaning of $\alpha<\beta$ is clear and, in fact, $(\{\alpha: \alpha<\beta\},<)$ is of order type $\beta$. A cardinal number $\lambda$ is identified with $\{\alpha: \alpha$ is an ordinal and $|\{\beta: \beta<\alpha\}|<\lambda\}$, a set of cardinality $\lambda$; this is the representative.

We denote by $\aleph_{\alpha}$ the $\alpha$-th infinite cardinal (so GCH means $2^{\aleph_{\alpha}}=\aleph_{\alpha+1}$ ).

\section{$\S 1$ What Does MATHEMATICAL LOGIC DO FOR YOU?}

What does mathematical logic deal with? It is mathematics applied to mathematics itself (and to some problems in philosophy).

But what does it do for you, a mathematician from another field? It does not help you to solve a thorny differential equation or anything like that. But if you suspect that the Riemann Hypothesis or $\mathrm{P}=\mathrm{NP}$ is undecidable or, say, cannot be decided by your present methods by whatever ingenuity which can be mastered, then you are on the hook: how can you even phrase this coherently, let alone prove anything like this?

For such questions you need to phrase a general framework for doing mathematics (this is set theory - usually axiomatized in the "ZFC set of axioms" 1 or relatives of it); of course set theory is far from being motivated just by this.

You also need to define what a mathematical proof is (Gödel's completeness theorem, as well as Gödel's incompleteness theorem, speaks about the relationship

\footnotetext{
${ }^{1}$ Axioms of Zermelo and Fraenkel with Axiom of Choice.
} 
between provability and truth; more on this than I would like to know is the subject of proof theory).

You may develop mathematical theories well enough without looking for mathematical logic, but if you like a formalization of "a mathematical theory" and consider investigation at such a level of generality, you arrive at model theory. You may wonder whether anything interesting can be said about such an arbitrary theory; does model theory just grind water or does it have theorems with meat? Does model theory pass this test? Certainly, I definitely think that it does. Does model theory have relevance to other parts of mathematics? I think that for a general theory to give interesting results when specialized to older contexts is strong evidence of its being deep (though certainly not a necessary condition). Naturally, I think that model theory passes this test too, and notions which arise from investigating the structure of a general class of structures in arbitrary cardinality later serve in investigating classical objects. Though I have much to say on model theory, I have not managed to write what I would like to say on it, so except for $\S 6$ the present paper does not deal with model theory, but only with set theory.

Similarly if you like to know about inherent limitations of algorithms and the semi-lattice of Turing degrees, you turn to computability (which used to be called recursion theory, but also on this I plead ignorance).

What is this animal "ZFC"?

Fundamental for us is ZFC. I feel it is not something esoteric for you. Rather, most mathematicians "do not know" of ZFC, just as Monsieur Jourdain in Molière's Bourgeois Gentilhomme "did not know" he was speaking in prose or in the same way that most of us freely use the law of the excluded middle, " $A$ or non- $A$ ", without any scruples, because we do not even notice that we are using it. Many times, we can read but not understand (mathematical writings). However, I guarantee you will understand, though not necessarily be able to read (including most of my audience in LA and unfortunately myself), the following paragraph:

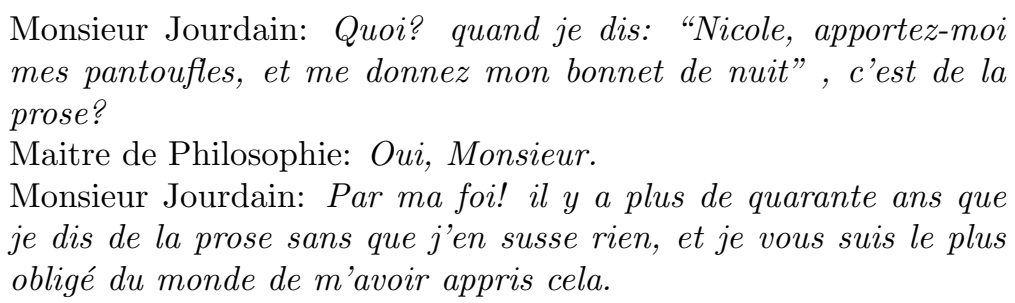

When I explain the axioms of ZFC, the usual response is "Fine, but by what right do you assume CH?" (the Continuum Hypothesis, see below, which is not included).

In non-technical terms,

1.1 The Jourdain Thesis. If you deal freely with the set of reals or the vector space of functions from the reals to the reals or, e.g., with $\operatorname{Hom}(G, H)$ for groups $G, H$, then you - for all practical purposes - work within and accept the axioms of ZFC; that is they formalize whatever proofs you accept. Even other positions (like omitting the axiom of choice or replacing it by weak versions) are best dealt with within this framework.

1.2 The Generality Thesis. If you would like to have general results, you have to use a set-theoretic framework. While by now we know well how to generate "generalized nonsense" which grinds water and tells us nothing new, many times a 
general framework shows you that isolated claims are parts of general phenomena; the real test for me is whether you discover deep, beautiful mathematics.

That is, if you want to know something about ALL structures of some kind (all groups, all manifolds, etc.), then you need to be able to deal with infinite unions or infinite products of sets, which are inherently set-theoretical concepts.

Moreover, even if your main interest is in, say, finitely generated groups, you will be drawn into more general ones, e.g., taking some compactification or using infinite products.

How far should you go? When to stop? I suggest that we adopt the following position:

1.3 The Jeffersonian Thesis. The best framework, the best foundation, is the one that governs you least; that is you do not notice its restrictions (except when really necessary, like arriving at a contradiction).

For instance, look at the following scheme of a proof: given a colouring of the natural numbers with red and green, we look at the Stone-Čech compactification $\beta(\mathbb{N})$ of $\mathbb{N}$, which is just the set of ultrafilters on $\mathbb{N}$ (the principal ultrafilters are identified with the natural numbers); we define on it an operation + :

$$
p+q=\{A \subseteq \mathbb{N}:\{n: n+A \in p\} \in q\},
$$

and using an easy fixed point theorem in the semi-group $(\beta(\mathbb{N}),+)$ we get an infinite monochromatic set such that its members and all (finite non-trivial) sums of members (with no repetition) have the same color (this is well known and gives a very elegant alternative proof of Hindman's theorem). Would you object to such a proof? Or would you stop at the power set $\mathcal{P}(\mathbb{N})$ of $\mathbb{N}$ ? First, it seems to me unnatural not to have $\mathcal{P}(\mathcal{P}(\mathbb{N}))$, and second you will not gain much; e.g., the problems of cardinal arithmetic are replaced by relatives even if you consider only cardinalities of sets of reals (on pcf theory see $\S 5$; also definable sets may give such phenomena).

There is a natural scale of theories, some stronger than ZFC (large cardinals), some weaker (e.g., PA = Peano Arithmetic $=$ a set of axioms for the natural numbers in first order logic describing addition, multiplication, and the scheme of mathematical induction).

PA already tells us that the universe is infinite, but PA "stops" after we have all the natural numbers. ZFC goes beyond the natural numbers; in ZFC we can distinguish different infinite cardinalities such as "countable" and "uncountable", and we can show that there are infinitely many cardinalities, uncountably many, etc.

But there are also set theories stronger than ZFC, which are as high above ZFC as $\mathrm{ZFC}$ is above PA, and even higher.

1.4 The Scale Thesis. Even if you feel ZFC assumes too much or too little (and you do not work artificially), you will end up somewhere along this scale, going from PA to the large cardinals. (What does "artificial" mean? For example: the theory "ZFC $+(\mathrm{a})+(\mathrm{b})+(\mathrm{c})$ " where the statements $(\mathrm{a}),(\mathrm{b}),(\mathrm{c})$ are:

(a) there are 17 strongly inaccessible cardinals,

(b) the theory ZFC + "there are 84 strongly inaccessible cardinals" is contradictory (this is a statement about arithmetic), and

\footnotetext{
${ }^{2}$ That is, an uncountable cardinal $\lambda$ satisfying $\mu<\lambda \Rightarrow 2^{\mu}<\lambda$ and the condition if $\lambda_{t}<\lambda$ for $t \in I$ where $|I|<\lambda$, then $\sum_{t \in I} \lambda_{t}<\lambda$; in other words, it is strong limit and regular.
} 
(c) the theory ZFC + "there are 49 strongly inaccessible cardinals" is consistent but has no well founded countable model.)

An extreme skeptic goes "below PA"; e.g., (s)he may doubt not only whether $2^{n}$ (for every natural number $n$ ) necessarily exists but even whether $n^{[\log n]}$ exists. (In the latter case (s)he still has a chance to prove "there are infinitely many primes".) The difference between two such positions will be just where they put their belief; so the theory is quite translatable, just a matter of stress. For instance, by one we know that there are infinitely many primes; by the other we have an implication. There is a body of work supporting this, the so-called equi-consistency results (e.g., on real valued measurable cardinals; more later).

So far I have mainly defended accepting ZFC; as for believing in more, read on.

$\S 2$ The glory of Proven ignorance: To show that we Cannot Know!

In short: The Continuum Problem asks: How many real numbers are there?

G. Cantor proved: There are more reals than rationals. (In a technical sense: "uncountable", "there is no bijection from $\mathbb{R}$ into $\mathbb{Q}$ ".)

The Continuum Hypothesis $(\mathrm{CH})$ says: Yes, more, but barely so. Every set $A \subseteq \mathbb{R}$ is either countable or equinumerous with $\mathbb{R}$.

K. Gödel proved: Perhaps CH holds.

P. Cohen proved: Perhaps $\mathrm{CH}$ does not hold.

What is a better starting point than Hilbert's first problem, the Continuum Problem? It deals with the arithmetic of infinite numbers, called cardinals, discovered by Cantor; they are just the equivalence classes of the relation "there is a one-to-one mapping from $A$ onto $B$ " with natural operations. Specifically, it asks whether the continuum, the number of reals, is the successor of the number of natural numbers. In other words, can we for any infinite set of real numbers find a one-to-one map from it onto the set of all reals or onto the set of natural numbers? I find this a great problem; in particular it has induced much of the 20th century achievements in set theory.

Gödel has shown that the Generalized Continuum Hypothesis (GCH) may hold. In fact, it holds if we restrict ourselves to the class of the constructible sets: the universe $\mathbf{L}$ consisting of the constructible sets, which satisfies all the axioms of set theory and also GCH (see §3). The class $\mathbf{L}$ can be described as the minimal family of sets you have to have as long as you have the same ordinals (that is, order types of linear orders which are well founded, i.e., linear orders for which every nonempty set has a first element). On the other hand, Cohen has proved that you cannot prove the Continuum Hypothesis. Whereas Gödel has "shrunk" the universe, Cohen has "extended" it, adding "generic" subsets to old partially ordered sets (which from this perspective are called forcing notions).

Gödel: CH cannot be refuted. Moreover, the Generalized Continuum Hypothesis may hold; in fact it holds if we restrict ourselves to the class $\mathbf{L}=$ the class of constructible sets.

Cohen: You cannot prove that all sets are constructible, and you cannot even prove the weaker statement $\mathrm{CH}$.

Cohen discovered the method of forcing and used it to prove this "independence" result. 
So the history of set theory is like that of many people: first non-self-awareness, the happiness of discovering the world (not paying too much attention to B. Russell), then going on a crash diet for a look of thinness ( $\mathbf{L}$ of Gödel), and then discovering the comforts of growing a nice belly (after Cohen).

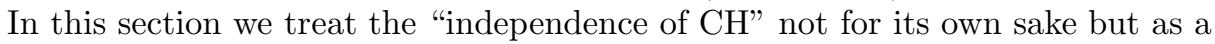
prototype for an independence result (= proof of unprovability).

2.1 Dream. 1) Find a "forcing method" relative to PA which shows that PA and even ZFC does not decide "reasonable" arithmetical statements, just like the known forcing method works for showing that ZFC cannot decide reasonable set-theoretic questions. Even showing the unprovability of various statements in bounded arithmetic (instead of PA) is formidable.

2) In particular, in a way parallel to forcing, find independence results for classical problems in number theory; for example:

$(\square)$ prove that various statements formalizing the "randomness of the prime numbers" (e.g., there are infinitely many primes of the form $n^{2}+1$; see below) are independent (from PA and even ZFC and other such statements).

Of course, $(\square)$ is a possible materialization of

2.2 Dream. Find a theory which will formalize the thesis that the primes behave like a random set of natural numbers gotten by tossing a coin with probability $1 / \log (n)$ for deciding if $n$ is prime.

Of course a parallel achievement for other classical problems in number theory will be very welcome too.

Warning: There are two major known kinds of "independence results". The method of forcing can be used only to make the universe "fatter", not "taller". In technical terms: if we use forcing starting from models of ZFC to prove that "ZFC neither proves nor refutes statement A" (or equivalently, each of "ZFC + $\mathrm{A}$ " and "ZFC + non-A" is consistent), then the "consistency strengths" of "ZFC", "ZFC+A", "ZFC + non-A" are all equal.

We should not confuse forcing with another method for proving independence, that of consistency strength. This means sentences like those gotten by Gödel's incompleteness theorem and its many illustrious descendants, some of which are discussed below; for example:

"In ZFC we cannot prove that ZFC is consistent."

(See more in the section after 3.1.) Here we do not use forcing; rather we rely on the fact that the consistency strength of "ZFC + ZFC is consistent" is strictly higher than the consistency strength of ZFC alone.

Independence results (=unprovability proofs) of this second kind (comparing consistency strengths) are possible also over much weaker theories such as Peano Arithmetic, a common axiomatization for the natural numbers.

Note that if we take as our base PA instead ZFC, statements like "every definition $\{x \in \mathbb{N}: \varphi(x)\}$ defines a set" play the role of large cardinals.

There has been much work devoted to trying to find finitary combinatorial statements which are equiconsistent with such "large cardinal statements": Paris and Harrington [PH77], Kirby and Paris [KP82] (the Hydra), Ketonen and Solovay KS81, and many works of Harvey Friedman; see [FOM for much information and

discussion. Friedman has been saying for some years that all this is irrelevant to 
"mainstream mathematics" and thinks that other mathematics relevant to his discoveries will be developed. So from his view it follows that Dream 2.3 below is false.

Let me stress that those two methods of forcing and of comparing consistency strength are incomparable; though both may sometimes prove the same theorem, they say different things: the forcing one is more specific to set theory, gives a strong "no answer", whereas the method of comparing consistency strength (or large cardinals) gives an answer whose meaning may naturally lead to debate. It depends on our degree of confidence in the consistency of the large cardinal considered. Also it has a limitation as a method of proving independence: if $\mathrm{ZFC}+\varphi_{1}$ is equiconsistent with ZFC + " $\exists$ a strongly inaccessible cardinal" whereas ZFC $+\varphi_{2}$ is equiconsistent with $\mathrm{ZFC}$, then $\mathrm{ZFC}+\varphi_{2}+\neg \varphi_{1}$ is consistent, but we cannot do anything about $\mathrm{ZFC}+\varphi_{1}+\neg \varphi_{2}$.

My "dream" about classical problems in number theory refers to independence results of the first kind, such as those obtained by forcing, where consistency strength is not increased. Concerning the other method:

2.3 Dream. Prove that the Riemann Hypothesis is unprovable in PA but is provable in some higher theory.

What basis does my hope for this dream have? First, the solution of Hilbert's 10 th problem tells us that each problem of the form "is the theory ZFC $+\varphi$ consistent" can be translated to a (specific) Diophantine equation being unsolvable in the integers; moreover the translation is uniform (this works for any reasonable (defined) theory, where consistent means that no contradiction can be proved from it). Second, we may look at parallel development "higher up", as the world is quite ordered and reasonable.

Note that there is a significant difference between $\Pi_{2}$ sentences (which say, e.g., for a given polynomial $f$, the sentence $\varphi_{f}$ saying that for all natural numbers $x_{0}, \ldots, x_{n-1}$ there are natural numbers $y_{0}, \ldots, y_{m}$ such that $\left.f\left(x_{0}, \ldots, y_{0}, \ldots\right)=0\right)$ and $\Pi_{1}$ sentences saying just that, e.g., a certain Diophantine equation is unsolvable. The first ones can be proved not to follow from PA by restricting ourselves to a proper initial "segment" of a nonstandard model of PA. For $\Pi_{1}$ sentences, in some sense proving their consistency shows that they are true (as otherwise PA is inconsistent). Naturally, concerning statements in set theory, models of ZFC are more malleable, as the method of forcing shows.

2.4 Problem. Show that forcing is the unique method in a deep sense.

I have the impression that number theorists were generally not so excited about the unsolvability of Diophantine equations. Probably they reasoned that they did not hope for such a grand solution anyway and all this has no direct bearing on their work. So far they are right, but several times researchers in other fields have felt similarly (true, in a posteriori wisdom we can explain the difference, but we all do not lack this kind of wisdom); clearly my dream contradicts this feeling.

\section{$\S 3$ Set theory and additional axioms}

Gödel's sentences and large cardinals:

So the sentence saying "There is no proof of contradiction in PA (Peano Arithmetic)" or similarly in ZFC is undecidable in this theory (but in ZFC we can prove the consistency of PA). Now it seems strange to believe in PA while not believing 
in "PA+ $\mathrm{CON}(\mathrm{PA})$ " where $\mathrm{CON}(\mathrm{T})$ is the assertion that the theory $T$ is consistent (but where to stop?), so though we prove independence, this is not like the proof of the independence of $\mathrm{CH}$, which leaves us with no indication of what is true; a related difference is also expressed by "those sentences are metamathematical". Related to this incompleteness method are large cardinals. The so-called "large cardinal axioms" state that a "large cardinal $\kappa$ " exists, which means it resembles $\aleph_{0}$ in some sense, in particular with properties implying that $\mathcal{H}(\kappa)$, the family of sets with hereditary closure of cardinality $<\kappa$, form a model of ZFC and more. Noticeable among them is " $\kappa$ is a measurable cardinal", which says that there is a $0-1$ measure on the family of subsets of $\kappa$ which gives singletons measure zero and is $<\kappa$-complete (that is, the union of $<\kappa$ sets of measure zero is of measure zero). The first large cardinal property is " $\kappa$ is a strongly inaccessible cardinal", which means that it is strong limit (i.e., $\mu<\kappa \Rightarrow 2^{\mu}<\kappa$ ), regular (i.e., is not the sum of $<\kappa$ cardinals each $<\kappa$ ) and uncountable (the other two properties are enjoyed by $\aleph_{0}$ ). I feel those are mathematical statements in set theory; some others will call them non-mathematical, but logical or set-theoretical. They reserve "mathematical" for number theory or generally what they call "mainstream mathematics".

Strengthening ZFC, new axioms:

Should we add more axioms to ZFC? There were some options on various grounds. For some time using GCH was quite popular; it gives a coherent theory for set theory of the real line (e.g., there is an uncountable set $S$ of reals such that every uncountable subset of $S$ is not of Lebesgue measure zero (or is not meagre); see, e.g., the writings of W. Sierpiński). It was also used extensively in the partition calculus investigation (see Erdös, Hajnal and Rado [EHR]) and in model theory in the 1950's and 60's: e.g., in the use of saturated models and in Keisler's theorem that every two elementary equivalent models have isomorphic ultrapowers.

It became reasonable to assume GCH (and clearly if you prove a statement using ZFC + GCH, then you cannot refute in ZFC that statement, by Gödel's work on L). Some people look at this behaviour as adopting GCH, and it could be argued that you do not lose much (e.g., ZFC and ZFC + GCH have the same arithmetical consequences). Still most mathematicians, even those who have worked with GCH, do it because they like to prove theorems and they could not otherwise solve their problems (or get a reasonable picture); i.e., they have no alternative in the short run. Clearly, even after forcing was found, it seems better to prove that something follows from GCH than just proving it is consistent; statements which we treat like this we shall call semi-axioms (later we explicate). Of course, the extent to which we consider a statement a semi-axiom is open to opinion and may change in time. I give statements in cardinal arithmetic a high score in this respect.

Note that a semi-axiom may be (consistent with ZFC and) very atypical (= the family of universes satisfying it is "negligible") but still very interesting, since for some sets of problems it gives a nice coherent picture.

After the works of K. Gödel and R. B. Jensen on $\mathbf{L}$, when it became clear that there is very little independence over $\mathrm{ZFC}+\mathbf{V}=\mathbf{L}$, adopting $\mathbf{V}=\mathbf{L}$ as an axiom became an issue. It is useful, it decides many problems (in particular it implies GCH so we understand cardinal arithmetic), it also is a natural statement. Just one problem: Why the hell should it be true? (What does true mean here? See below.) Jensen has thought that to prove a statement holds in $\mathbf{L}$ or just in a universe which is canonical or a universe which has a "structure" (a parallel of his 
fine structure theory) is better than just proving consistency. I think so too and give $\mathbf{V}=\mathbf{L}$ and other inner models semi-axiom status, though probably less high than Jensen does.

J. Kennedy wondered what I mean by "V $=\mathbf{L}$ is not true".

Some believe that compelling, additional axioms for set theory which settle problems of real interest will be found or even have been found. It is hard to argue with hope and problematic to consider arguments which have not yet been suggested. However, I do not agree with the pure Platonic view that the interesting problems in set theory can be decided, that we just have to discover the additional axiom. My mental picture is that we have many possible set theories, all conforming to ZFC. I do not feel "a universe of ZFC" is like "the Sun", it is rather like "a human being" or "a human being of some fixed nationality"; see more in [Sh:E16].

So my meaning in saying "why the hell should it be true?" is not that it is provably false, just as "the national lottery in the last ten years was won successively in turn by the nephews of the manager, so we know that there was cheating" is mathematically not proved. Clearly $\mathbf{L}$ is very special, to some extent unique; thus, the statement $\mathbf{V}=\mathbf{L}$ should get probability zero (thought not being impossible). So $\mathbf{L}$ is certainly a citizen with full rights but a very atypical one. Also a typical citizen will not satisfy $(\forall \alpha)\left[2^{\aleph_{\alpha}}=\aleph_{\alpha+\alpha+7}\right]$ but probably will satisfy $(\exists \alpha)\left(2^{\aleph_{\alpha}}=\aleph_{\alpha+\alpha+7}\right)$. However, some statements do not seem to me clearly classified as typical or atypical. You may think "Does $\mathrm{CH}$, i.e., $2^{\aleph_{0}}=\aleph_{1}$ hold?" is like "Can a typical American be Catholic?" More reasonably, $\mathrm{CH}$ has a small measure, but still much, much more than $\mathbf{V}=\mathbf{L}$. For set theorists I will add that $\exists 0^{\#}$ is for me a candidate for a statement with positive measure and with a positive measure for its negation.

What about ZFC + "ZFC is inconsistent"? Clearly we would not consider such theories, except that Gödel's incompleteness theorems force us to do it. So if $\mathbf{V}=\mathbf{L}$ is an atypical citizen, such a theory should perhaps be a permanent illegal immigrant.

But I am very interested in

3.1 Dream. Find statements which yield a wonderful theory, so will therefore be accepted as additional semi-axioms in the sense above, not just as other cases of those above.

Large cardinals are certainly natural statements, as their role in finding a quite linear scale of consistency strength on statements (arising independently of them) shows. But there is an important group with stronger beliefs: the California school of set theory, which holds that $\mathrm{AD}_{\mathbf{L}[\mathbb{R}]}$ (and relatives which we explain below) is true. They are interested mainly in descriptive set theory, so let me first try to explain it. The point is that we know that some sets of reals are not Lebesgue measurable, but Borel sets are, and so are $\Sigma_{1}^{1}$-sets (projections to $\mathbb{R}$ of Borel subsets of $\mathbb{R} \times \mathbb{R}$ ). But this is not necessarily true for projective sets (= the sets belonging to the closure of the family of Borel subsets of the $\mathbb{R}^{n}$ 's under projection and complement).

In particular if $\mathbf{V}=\mathbf{L}$, this is false (i.e., not all projective sets of reals are Lebesgue measurable), and the California school believes that generally it gives a "false", uninteresting picture and that the answers we get in this case are "incidental" and "artificial". This is very reasonable. For them, the statement $\mathrm{AD}_{\mathbf{L}[\mathbb{R}]}$, and lately the star-axiom from Woodin Wd00, are the remedies they adopt. Just as $\mathrm{AD}_{\mathbf{L}[\mathbb{R}]}$ solves "correctly" the theory of $\left(\mathcal{H}\left(\aleph_{1}\right), \in\right)$, so does the star-axiom solve 
correctly the problem of $\left(\mathcal{H}\left(\aleph_{2}\right), \in\right)$. In particular, $\mathrm{AD}_{\mathbf{L}[\mathbb{R}]}$ implies that all projective sets of reals are Lebesgue measurable and generally give the "right theorems of descriptive set theory." The star-axiom gives much more; in particular it implies the continuum, i.e., the number of reals is $\aleph_{2}$.

The argument of the California school for $\mathrm{AD}_{\mathbf{L}[\mathbb{R}]}$ is that it gives a coherent, true picture for a group of problems on the continuum, e.g., the behavior of the projective sets, and implies that the natural problems in the theory of projective sets of reals are decidable and in the "right way". Also, such axioms (e.g., $\mathrm{AD}_{\mathbf{L}[\mathbb{R}]}$ ) are equi-consistent with a suitable large cardinal; moreover such axioms follow from the existence of the suitable large cardinal.

Now the California school does not have an accepted catechism, different members may differ, and even the same reasonable person may vary according to time or place, but I heard many times that " $\mathrm{AD}_{\mathbf{L}[\mathbb{R}]}$ is true". More specifically, for definitiveness, let me quote two major opinions. H. Woodin, in a seminar at the Mittag-Leffler Institute (Fall 2000), says that the work on problems on implications between variants of the axiom of choice (so prominent in set theory till the sixties) has been essentially deserted, marginalized since the Axiom of Choice is just accepted as true. Similarly, he thinks the star-axiom will be accepted as true and research which was done on problems it answers by other approaches will be marginalized. In an informal symposium in Väänänen's apartment around the same time, after I explained my position on very interesting semi-axioms, J. Steel persuasively argued, "So having agreed that not all set theories are equal, some are more interesting, they are better, so make one more step, is there not the best ... (star-axiom)?" Woodin Wd00 is an excellent presentation and is written in a non-polemic style.

Let me stress that the mathematics of the California school is great: deep and interesting; the program succeeded in discovering a major semi-axiom. Unlike Woodin, I feel that probably the right analogy is between the status of $\mathrm{AD}_{\mathbf{L}[\mathbb{R}]}$ and the star-axiom now with that of $\mathrm{GCH}$ and $\mathbf{V}=\mathbf{L}$ earlier. At the time they were much more informative than any alternative; now they are not marginalized, just not "the favorite son".

I strongly reject the California school's position on several grounds.

(a) Generally I do not think that the fact that a statement solves everything really nicely, even deeply, even being the best semi-axiom (if there is such a thing, which I doubt) is a sufficient reason to say it is a "true axiom". In particular I do not find it compelling at all to see it as true.

(b) The judgments of certain semi-axioms as best is based on the groups of problems you are interested in. For the California school, descriptive set theory problems are central. While I agree that they are important and worth investigating, for me they are not "the center". Other groups of problems suggest different semi-axioms as best; other universes may be the nicest from a different perspective.

(c) Even for descriptive set theory the adoption of the axioms they advocate is problematic. It makes many interesting distinctions disappear (see more below).

Now I reject also the extreme formalistic attitude which says that we just scribble symbols on paper or all consistent set theories are equal (see above before 3.1). 
3.2 Dream. Find universes with a different but interesting theory of projective sets (i.e., universes in which $\mathrm{AD}_{\mathbf{L}[\mathbb{R}]}$ fails).

Note that $\mathbf{V}=\mathbf{L}$ gives a very clear theory but one where, e.g., we have a projective well ordering of reals (hence a non-Lebesgue-measurable set). Of course, $\mathrm{AD}_{\mathbf{L}[\mathbb{R}]}$ is much more reasonable than the star-axiom, which is very special, though not as special as $\mathbf{V}=\mathbf{L} ; \mathbf{V}=\mathbf{L}$ has probability zero, whereas $\mathrm{AD}_{\mathbf{L}[\mathbb{R}]}$ has higher "probability", but it excludes no less interesting universes. Even for set theory of the reals, the possibility of the continuum being real valued measurable is not less important (more on it later; anyhow it implies that the continuum is large).

So according to the view presented here, $\mathrm{AD}_{\mathbf{L}[\mathbb{R}]}$ is certainly a semi-axiom: a beautiful theory with fascinating theorems built on it. I may even agree it has "positive measure" and is not atypical. But "there is no inner model with suitable large cardinal" seems to me also of positive measure. Also the large cardinals themselves seem to me of positive, co-positive measure (decreasing with largeness, of course).

I think $\mathbf{V}=\mathbf{L}$ passes this criterion of being a great semi-axiom for many problems. It is extremely helpful in building examples; e.g., it gives a very coherent theory on an important group of problems in Abelian group theory (see Eklof and Mekler [EM], [EM02]).

The descriptive set theorists have reasonable reasons to reject the axiom $\mathbf{V}=\mathbf{L}$, but are there not similar grounds for rejecting the continuum being $\aleph_{n}, n$ a natural number, hence rejecting the star axiom? Various combinatorial properties of the continuum follow from $2^{\aleph_{0}}=\aleph_{n}$ which are akin to having a definable well order (on $(n+1)$-place function from reals to reals); see [EHR]. Moreover, this applies even to problems with descriptive set theory flavours. For example, I am currently interested in a very explicit definition of an Abelian group $G_{n}$ (for $n$ a natural number; we can represent the set of elements as the reals, and $x \in G, x+y,-x$ are not just Borel but even $F_{\sigma}$ ). Now this Abelian group is free iff $2^{\aleph_{0}}<\aleph_{n+1}$ (see Sh 771 §5]). It seems to me that its being free is accidental, just that the continuum is not large enough for us to see how complicated it is, to see its true nature. So assuming $2^{\aleph_{0}} \leq \aleph_{n}$ is artificial is wrong, just as $\mathbf{V}=\mathbf{L}$ is wrong for "are projective set Lebesgue measurable".

It may be more interesting to consider a family of problems on the continuum: investigate cardinal invariants. We may measure the continuum not only in size but in other ways, like non(null) $=$ the minimal cardinality of a non-null set or non(meagre) $=$ the minimal cardinality of a non-meagre set (= not first category set), $\mathfrak{d}=\{|\mathcal{F}|: \mathcal{F}$ a family of functions from $\mathbb{N}$ to $\mathbb{N}$ such that every such function is bounded by one of them $\}$. There is a myriad of such measures, many of them important in many directions (see Bartoszyński [Baxx], Blass [Bsxx]); naturally they are uncountable but $\leq 2^{\aleph_{0}}$.

If the continuum is $\leq \aleph_{2}$, we will not have (by a trivial pigeonhole principle) many relations, as no three can be simultaneously distinct. This seems to me no less artificial than the answers to descriptive set-theoretic problems in $\mathbf{L}$.

3.3 Dream. Find a consistent axiom which gives a coherent true picture for cardinal invariants of the continuum; i.e., it implies that any two cardinal invariants which are "not necessarily equal" will not be equal, so necessarily the continuum is large (as there are many such invariants; see Goldstern and Shelah [GoSh 448]). 
Such an axiom is not necessarily unique, since for many pairs of cardinal invariants both inequalities are consistent.

This is naturally connected to

3.4 Dream. 1) Develop a theory of iterated forcing for a large continuum as versatile as the one we have for $2^{\aleph_{0}}=\aleph_{2}$ and, to a lesser extent, for $2^{\aleph_{0}}=\aleph_{1}$ (see [Sh:f] and a different approach in Woodin [Woxx]), but see 5.3.

2) So far we have many independence results and few theorems, but hopefully (see the rubble thesis in $\S 5$ ) as in the case of cardinal arithmetic a positive theory will appear.

Another approach, not disjoint to the descriptive set theory one, is to adopt large cardinal axioms, the argument being that they are the natural extension of how we arrive at ZFC.

A large cardinal axiom is one stating there is an uncountable cardinal $\lambda$ which satisfies a property satisfied by $\aleph_{0}$ like "strongly inaccessible"; see above. A picture justifying the existence of a large cardinal is defining by induction on the ordinal $\alpha$ the set $\mathbf{V}_{\alpha}$ by $\mathbf{V}_{0}=\emptyset, \mathbf{V}_{\alpha}=\bigcup\left\{\mathcal{P}\left(\mathbf{V}_{\beta}\right): \beta<\alpha\right\}$. The intuitive argument is that if we "wait long enough", i.e., for some $\alpha$ large enough, whatever is not forbidden to happen will happen, so there are $\alpha$ which reflect well what the whole universe $\mathbf{V}=\bigcup\left\{\mathbf{V}_{\alpha}: \alpha\right.$ an ordinal $\}$ satisfies. This seems to me reasonable (but see later). A motivation was the hope that this would decide classical problems about relatively small sets; it has failed for $\mathrm{CH}$ (though above a supercompact cardinal, cardinal arithmetic is simple) but has succeeded for problems like "every projective set of reals is Lebesgue measure" (see above). In my view the analogy of arriving at large cardinals with ZFC is problematic: we arrive at ZFC by considering natural formations of sets (the set of natural numbers, taking Cartesian products and power sets); even the first strongly inaccessible cardinal has no parallel justification. If you go higher up in the large cardinal hierarchy, the justification for their existence decreases, so large cardinal axioms are great semi-axioms but not to be accepted as true.

J. Kennedy has wondered where do we make the comparison between various set theories and by what criterions. Now we can do it informally; Cantor understood set theory quite well and understood the Continuum Problem without sticking to a formalization. Alternatively, we may work within a "bare bones set theory", just enough to formalize first order theories, proofs, and, say, having the completeness theorem. We may well agree we are in a universe which is set theory and discuss it without a priori having a common agreement on all its properties.

What are our criterions for semi-axioms? First and most important, it must have many consequences, making it have a rich, deep, beautiful theory. Second, it is preferable that it is reasonable and "has positive measure". Third, it is preferred that it leads to no contradiction (so lower consistency strength is better).

Naturally, these are conflicting hopes. So $\mathbf{V}=\mathbf{L}$ is preferable to GCH, as it has more consequences; but as $\mathrm{GCH}$ is much more reasonable and still has a very large set of consequences, it is worthwhile to use it and try to prove from it what earlier was proved from $\mathbf{V}=\mathbf{L}$.

Is the position presented here consistent? Can I on the one hand be opposed to "the true, unique set theory" and on the other hand not give equal weight to all consistent set theories (measure zero to $\mathbf{V}=\mathbf{L}$ )? Having pointed out a case where we accept such a position (typical American), I think I have shown the consistency 
of this position. In fact, considering any biography of a person is no better than the "typical American", as any interesting statement about a person normally has such character; we may well say "X is normally cheerful, but today is sad."

It may help the reader to note that my interest in mathematical logic has been mathematical, seeking generality, rather than philosophical. So we have not seriously addressed issues which do not seem to make a difference to mathematics: you may be a pure Platonist, telling me that there is a unique universe of set theory about which we know no more than ZFC, or that you believe in going to the sources ("would Cantor have such a thing in mind?") or that you believe that the axioms of set theory reflect the relationship between the two hemispheres of the human brain or that we discover (rather than invent) it by our research or that you may think that starting with some mental picture, we change it as our knowledge increases and converge to a "natural theory" (up to presentation). Very important and fascinating intellectual issues, but they will not make a real difference to the discussion here.

\section{$\S 4$ ZFC INDECISIVENESS}

Again, I certainly think large cardinals are natural notions, an indispensable part of our knowledge. For me the most compelling reason for their being important is the linear order phenomena and their roles in equi-consistency; see below.

Two statements $\varphi, \psi$ are equi-consistent if we can prove (even in PA) that ZFC $+\varphi$ is consistent iff $\mathrm{ZFC}+\psi$ is consistent; i.e., ZFC $\models \neg \varphi$ iff $\mathrm{ZFC} \models \neg \psi$. Now:

(A) For a plethora of classical problems $\varphi$ in set theory, large cardinal statements $\varphi_{L C}(x)$ were found such that $\varphi$ and $\exists \kappa \varphi_{L C}(\kappa)$ are equi-consistent or at least were sandwiched between two large cardinal statements. In fact, for most of the cases, if set theorists were really interested, they could get equi-consistency results; for examples see below.

$(B)$ The large cardinals are linearly ordered; i.e., for two such properties $\varphi_{L C}^{1}(\kappa)$, $\varphi_{L C}^{2}(\kappa)$ we can almost always prove for some $\ell \in\{1,2\}$ that

$\left(\square_{\varphi_{L C}^{1}, \varphi_{L C}^{2}}\right) \operatorname{CON}\left(\mathrm{ZFC}+\exists \kappa \varphi_{L C}^{\ell}(\kappa)\right)$ implies CON $\left(\mathrm{ZFC}+\exists \kappa \varphi_{L C}^{3-\ell}(\kappa)\right)$.

That is, relative consistency gives a linear order (transitivity holds trivially; the interesting phenomena is the comparability).

In fact, usually when $\left(\square_{\varphi_{L C}^{1}, \varphi_{L C}^{2}}\right)$ holds, the result is more explicit: if $\kappa_{2}$ satisfies $\varphi_{L C}^{2}(-)$, then we can find $\kappa_{1}<\kappa_{2}$ such that $\varphi_{L C}^{1}\left(\kappa_{1}\right)$ (at least in a smaller universe).

The way in which we prove the consistency of ZFC $+\varphi$ from the consistency of $\mathrm{ZFC}+\varphi_{L C}(\kappa)$ is usually by forcing. The way in which we prove the consistency of $\mathrm{ZFC}+\varphi_{L C}(\kappa)$ from the consistency of $\mathrm{ZFC}+\varphi$ is by inner models like $\mathbf{L}$ (i.e., we shrink the universe of sets, putting in only "necessary sets").

4.1 Dream. Explain the phenomena of linearity of consistency strength (and of large cardinal properties): to what extent is it the outcome of the history of set theory, the history of mathematics and human perceptions? Of course, we hope this will lead to exciting mathematical discoveries.

We may give a naive answer along the following lines:

For a large cardinal statement $\varphi=\varphi_{L C}(\kappa)$, let $\alpha_{\varphi}=\operatorname{Min}\{\alpha: \alpha$ is a countable ordinal, and there is a countable set $\mathbf{V}^{*}$ which is transitive (i.e., $x \in y \in \mathbf{V}^{*} \Rightarrow x \in$ $\left.\mathbf{V}^{*}\right)$, and an ordinal is in $\mathbf{V}^{*}$ iff it is $<\alpha$, and $\mathbf{V}^{*}$ is a model of ZFC $\left.+\exists \kappa \varphi(\kappa)\right\}$. 
So we actually investigate $\alpha_{\varphi^{1}} \leq \alpha_{\varphi^{2}}$, which is linear. But this does not explain why the statements we are interested in so far tend to be equi-consistent with such statements; we can produce counterexamples, but they are artificial (see [Sh 170, and lately I heard of recent lectures of Woodin). Like several other dreams, here it gets foggy: having an answer may well be disputable; it may have no solution or several.

As an illustration consider the classical case of real-valued measurable cardinals. We know that, unfortunately, we cannot have a measure on all sets of reals as good as Lebesgue measure, but mathematicians have tried to remedy this. One way is to omit the requirement that the measure $\mu$ be preserved by translations (so restricting the attention to the unit interval, any $A \subseteq[0,1]_{\mathbb{R}}$ is given a measure which is a real number $\mu(A) \in[0,1]_{\mathbb{R}}$, the measure is $\sigma$-additive (if $A_{n}$ are pairwise disjoint, then $\left.\mu\left(\bigcup\left\{A_{n}: n \in \mathbb{N}\right\}\right)=\sum\left\{\mu\left(A_{n}\right): n \in \mathbb{N}\right\}\right), \mu\left([0,1]_{\mathbb{R}}\right)=1, \mu(\emptyset)=0$ and $\left.\mu(\{x\})=0\right)$. If $\lambda=\operatorname{Min}\{|A|: \mu(A)=1\}$, we say that $\lambda$ is real-valued measurable since we can copy the measure from the set $A$ to $\lambda$.

Investigations on such measures on bigger sets lead to measurable cardinals; a cardinal $\lambda$ is called measurable if on some set $A^{*}$ of cardinality $\lambda$ there is a $\{0,1\}$ measure; i.e., $\mu: \mathcal{P}\left(A^{*}\right) \rightarrow\{0,1\}$ with $\mu(\emptyset)=0, \mu\left(A^{*}\right)=1, \mu(\{x\})=0$, which is even $(<\lambda)$-additive (i.e., if $A_{i} \subseteq A^{*}$ for $i \in I$ are pairwise disjoint and $|I|<\lambda$, then $\left.\mu\left(\bigcup\left\{A_{i}: i \in I\right\}\right)=\max \left\{\mu\left(A_{i}\right): i \in I\right\}\right)$. This is one of the most important large cardinal properties. In particular, its existence is unprovable in ZFC.

$\mathrm{R}$. Solovay has shown that if there is a measurable cardinal, then in some universe (obtained by a forcing extension) the continuum is real-valued measurable, thus showing the relative consistency. The reader may hope for a proof without a large cardinal or at least with a smaller large cardinal, but (s)he will hope in vain. Solovay proves that if there is a measure as above on the family of all subsets of $[0,1]_{\mathbb{R}}$, then if we shrink the universe (getting a so-called inner model) we can get a universe with a measurable cardinal. True, the two properties have some affinity to begin with, but this serves well as a measuring stick. To have $\mathrm{AD}_{\mathbf{L}[\mathbb{R}]}$ requires much larger cardinals; to have "all projective sets are Lebesgue measurable" requires much less. Moreover, this analysis leads sometimes to ZFC results; e.g., close to my heart is: if $A \subseteq[0,1]_{\mathbb{R}}$ has positive outer Lebesgue measure, then we can find pairwise disjoint sets $A_{n} \subseteq A$ for $n \in \mathbb{N}$, each with the same outer Lebesgue measure as $A$; see Gitik and Shelah GiSh 582.

Note that consistency strength gives us independence and more, hence necessarily is less versatile than forcing. Consistency strength can give one side of independence; forcing usually can give both, but, of course, the forcing may well start with large cardinals.

The notable hole in the program above is the supercompact, a very high, large cardinal which has been quite widely used in consistency proofs. The hole is that we do not know how to prove the consistency of ZFC + "there is a supercompact cardinal" from "low level statements".

4.2 Problem. Find an inner model for supercompactness. Without this we may always hope to replace it as an assumption for consistency results by smaller large cardinals (as had been done in some important cases).

The theory of forcing and, in particular, of iterated forcing seems important to me. There is a reasonable amount of such theory for forcing notions for the 
continuum; in the beginning we had one kind of such forcing: Cohen forcing. Now we know more, though far from what we like to know, as mentioned above. The case of continuum $>\aleph_{2}$ is a case where we miss much (in proof or in forcing, probably in both). For larger cardinals we know less; e.g., in work on the $2^{\mu}$ for $\mu$ singular, very sophisticated forcings were discovered (see writing of M. Gitik, M. Magidor, W. Mitchell and H. Woodin).

4.3 Question. Can we find a theory of iterated forcing parallel to the one we have for the continuum for the following purposes?

(a) For forcing as developed for the singular cardinal problem (see DjSh:659 ).

(b) For other cases (see more in [Sh 666]) on several missing theories of this kind.

4.4 Dream. Find additional methods for independence (in addition to forcing and large cardinals/consistency strength), or prove the uniqueness of these methods.

Dividing lines:

"Dividing line" or "watershed line" means here a property such that both it and its negation have strong consequences; hence it helps in proofs by cases. Dividing lines were fruitful in my work in model theory.

So $2^{\aleph_{0}}=\aleph_{\omega^{3}+\omega+1}$ does not look like a good dividing line (or even a property). What about $\mathrm{CH}$, i.e., $2^{\aleph_{0}}=\aleph_{1}$ ? This statement has many consequences and hence it is an impressive assertion, but its negation seemingly does not; so it is an important semi-axiom but not a good dividing line.

\subsection{Dream.}

(a) Find a real significance for $2^{\aleph_{0}}=\aleph_{753}$,

(b) or for $2^{\aleph_{0}}=\aleph_{\omega^{3}+\omega+5}$;

(c) show that all values of $2^{\aleph_{0}}$ which are $>\aleph_{2}$ are similar in some sense (or at least all values $\aleph_{n}>\aleph_{2}$, all regular $\aleph_{\alpha}>\aleph_{\omega_{1}}$ or whatever).

4.6 Dream. Can we find important dividing lines and develop a theory for combinatorial set theory?

Now Jensen has a different dream (I do not believe that it will materialize).

4.7 Dream. Find a super-duper "inner model" so large that basically it always behaves like $\mathbf{L}$ in a universe with no $0^{\#}$, so we can answer questions (in $\mathbf{V}$ ) by translating our problems to it, where we will have a fine structure to help us.

Of course, Jensen's optimism has something to do with his success with $0^{\#}$. If there is no such real, then the universe is "close to $\mathbf{L}$ "; otherwise it is very far from it.

Considering inner models, we find there are good dividing lines for descriptive set theory. Consider the statement "for every real $r, r^{\#}$ exists" (see, e.g., Jech [J]). Why is it a good dividing line? If $r$ is a counterexample, then descriptive set theory, the case where real parameters are allowed or just with parameter $r$, is very much like the one in $\mathbf{L}$, so all the questions traditionally asked are answerable, though, many say, in the "wrong, uninteresting" way. If it holds, we have determinacy for $\Sigma_{1}^{1}$ games (which gives nice consequences for "low level" projective sets of reals).

Let us consider two candidates for being dividing lines, unfortunately not impressive ones. The statement "there is a nice normal filter on $\omega_{1}$ " (see [Sh:g, Ch.V]) helps to prove cardinal arithmetic inequalities: If it holds, it is used to define rank 
on functions from $\omega_{1}$ to the ordinals. If it fails, the universe is similar enough to $\mathbf{L}$; hence cardinal arithmetic is trivial by Dodd and Jensen [DJ1] (see a use in ShSt 419]).

So far values of the continuum have not appeared to be good dividing lines, but we may look at another candidate. (Whereas $2^{\aleph_{0}}=\aleph_{1}$ has many consequences, $2^{\aleph_{0}}=\aleph_{2}$ has few consequences but many consistency results.)

The second candidate we consider is the cardinal arithmetic equality $2^{\mu}=\mu^{+}$, where $\mu$ is a strong limit singular cardinal, e.g., $\mu=\beth_{\omega}$; see $\S 5$.

Forcing for $\mathbf{L}$ : base theory = ZFC: highly developed "forcing" technology:

Forcing (as has been developed) was very successful to show that there are many problems which (like the continuum hypothesis) cannot be decided in set theory; moreover, this has become a method, which for a typical set-theoretic problem gives us a reasonable way to prove its consistency and its independence. This indicates that the axioms of set theory (ZFC) are weak, usually not able to decide given questions. However, those problems (such as $\mathrm{CH}$ ) which can be attacked by forcing can typically be decided by the single additional axiom that all sets are constructible, $\mathbf{V}=\mathbf{L}$. (Gödel proved that $\mathbf{L}$ is a model of $\mathrm{ZFC}+\mathrm{GCH}$. Jensen solved many specific problems and developed general methods under $\mathbf{V}=\mathbf{L}$.)

So this leads naturally to

4.8 Dream. Can we find a method parallel to forcing for $\mathbf{L}$ (i.e., for the usual axioms of set theory + every set is constructible)?

Such a method would enable us to prove that certain statements are independent of $\mathrm{ZFC}+\mathbf{V}=\mathbf{L}$; the current forcing results give only independence over ZFC.

A major preliminary obstacle to this dream is the lack of a good candidate to be a test problem, since so many questions have already been settled under the assumption $\mathbf{V}=\mathbf{L}$.

A negative answer, explaining why "a large body of set-theoretic problems is decidable" would be marvelous too (this would give "quasi-decidability" for $\mathbf{L}$ ).

\section{$\S 5$ IS ZFC REALLY SO WEAK?}

In this section we deal with a (relatively) new perspective on cardinal arithmetic.

5.1 The Not So Poor Thesis. The view that ZFC is a deficient theory, since it does not decide so many basic questions, in contrast to classical theories is one sided. In fact, a "random" question in, say, number theory is similarly hopeless as far as answerability is concerned, certainly practically, and by Gödel's incompleteness theorem + the undecidability of solvability of Diophantine equations, I think also fundamentally.

Set theory actually has an advantage over many other fields of mathematics. When we seem to be unable to prove or refute something, we have strong methods to try to show that a proof or refutation may be impossible. Such results, in addition to being interesting in their own right, also help to clear the air, directing us to what actually is decidable, discarding the undecidable ones. It also tells us what kind of problems are, at least relatively, of the answerable kind. 
5.2 The Rubble Removal Thesis. Methods for proving independence, in addition to their intrinsic value, work for us like a sieve: when we have a myriad of problems in some directions and we have tried to prove independence (and many times this results in discarding most of them), we are left with strong candidates for theorems of ZFC.

In this connection we may hope (compare with 3.3 ):

5.3 Dream. Our problem in proving consistency results for continuum $>\aleph_{2}$ comes from the existence of some positive theory which will become trivialized if the continuum is too small; i.e., most of the theorems which we will prove become trivialized.

The discussion in sections 2 and 4 may support the impression that "all is independent in ZFC"; this is not groundless but also not the whole truth. We shall now concentrate on cardinal arithmetic, not assuming specialized knowledge (see $\S 0$ for basic definitions).

For an even more leisurely explanation of cardinal arithmetic and pcf for the general mathematical audience, see [Sh:E25]; a book devoted to this subject is Sh:g].

Cardinal arithmetic is a good example for 5.2: after proving there is nothing more to say on $2^{\aleph_{0}}, 2^{\aleph_{1}}, 2^{\aleph_{\alpha+1}}$, we found that we can say something about $2^{\aleph_{\omega_{1}}}$ and even about $2^{\aleph_{\omega}}$ and about $\prod_{n<\omega} \aleph_{n}$. In fact, the thesis of [Sh:g] is that there are two separate phenomena. The first one is the behaviour of $2^{\lambda}$ for $\lambda$ regular (mainly $\lambda=\aleph_{0}, \aleph_{\alpha+1}$ ) for which everything is independent. The second one is the cofinality problem, the domain of pcf theory, which appears later.

In other words, looking at the bright side, we know all the true rules we can know for $2^{\lambda}$ for regular $\lambda$ (no more rules than the classical ones: it is non-decreasing, i.e., $\lambda \leq \mu \Rightarrow 2^{\lambda} \leq 2^{\mu}$ and $\operatorname{cf}\left(2^{\lambda}\right)>\lambda$; for cf see below). This leaves us with the singular cardinals like $\aleph_{\omega}=\sum\left\{\aleph_{n}: n\right.$ a natural number $\}$.

First it has been "clear" that the case of singular cardinals would be similar to that of the regular cardinals, just more complicated, a "technical problem". In fact, independence results were found for singular cardinals; however, using large cardinals. Second, it was proved that there are some limitations. Third, it was proved that large cardinals are necessary, using the theory of inner models (see Magidor [Mg1], [Mg2 for independence results; Silver [Si], Galvin and Hajnal [GH], [Sh 111, [Sh:b, Ch.XIII] on limitations on $2^{\lambda}$; Devlin and Jensen [DeJ], Dodd and Jensen [DJ1] on inner models; for more on forcing see the writings of M. Gitik, M. Magidor, W. Mitchell, H. Woodin; for more on inner models see the writings of M. Gitik, W. Mitchell, J. Steel).

A thesis of [Sh:g] is

5.4 Thesis. ["Treasures are waiting for you"] There are many laws of (infinite) cardinal arithmetic concerning exponentiation. In the past there seemed to be few and scattered ones because we concentrated on $2^{\lambda}$, but if we deal with a relatively small exponent and large base, there is much to be discovered; see more below.

Cardinal arithmetic investigations have concentrated on the function $\lambda \mapsto 2^{\lambda}$, for good reasons, but made us ignore other directions. Even after the independence results, researchers tended to be influenced by remnants of GCH, e.g., the concentration on $2^{\mu}$ for $\mu$ a strong limit singular cardinal. 
5.5 Dream. Find all the laws of (infinite) cardinal exponentiation.

pcf theory:

Close to my heart is

5.6 Thesis. Cardinal arithmetic is loaded with consistency results because we ask the wrong questions. The "treasures" thesis is not enough; we should replace cardinality by cofinality, as explained below (pcf theory).

5.7 Definition. 1) For a partially ordered set $P$ let $\operatorname{cf}(P)$, the cofinality of $P$, be $\min \left\{|Q|: Q \subseteq P\right.$ satisfies $\left.(\forall x \in P)(\exists y \in Q)\left[x \leq_{P} y\right]\right\}$.

2) $\operatorname{cf}\left([\lambda]^{\kappa}\right)=\operatorname{cf}\left([\lambda]^{\kappa}, \subseteq\right)$ is $\operatorname{cf}(\mathcal{P})$ when for some set $A$ of cardinality $\lambda, \mathcal{P}$ is the family of subsets of $A$ of cardinality $\leq \kappa$ partially ordered by inclusion.

3) We usually identify any cardinal $\lambda$ with a linear order, in fact a well ordering of this cardinality; every initial segment of it has smaller cardinality.

4) For a cardinal $\lambda$, let $\operatorname{cf}(\lambda)$ be $\operatorname{Min}\left\{|J|: \lambda=\sum_{t \in J} \lambda_{t}\right.$ for some $\lambda_{t}<\lambda$ for $t \in J\}$; this is compatible with the definition of $\mathrm{cf}$ in part (1). We call $\lambda$ regular if $\operatorname{cf}(\lambda)=\lambda$ and singular otherwise. Recall that $\aleph_{0}$ and every successor cardinal are regular, whereas $\aleph_{\omega}=\sum\left\{\aleph_{n}: n\right.$ a natural number $\}$ is the first singular cardinal, and $\operatorname{cf}(\lambda)$ is always a regular cardinal.

5) A partially ordered set $P$ is said to have true cofinality $\lambda$, and we write $\operatorname{tcf}(P)=\lambda$ if $(\lambda$ is a regular cardinal and $)$ there is a $\leq_{P}$-increasing sequence $\left\langle p_{i}\right.$ : $i<\lambda\rangle$ such that $(\forall q \in P)(\exists i)\left(q \leq_{P} p_{i}\right)$; note that $\lambda$ is unique. (Can $P$ fail to have true cofinality? Yes, e.g., if it is $P_{1} \times P_{2}$, ordered coordinate-wise, $P_{1}, P_{2}$ have true cofinalities but different ones.)

5.8 Convention. Let $\mathfrak{a}$ denote a set of regular cardinals $>|\mathfrak{a}|$; also let $\mathfrak{b}, \mathfrak{c}$ denote such sets.

5.9 Definition. 1) For an ideal $J$ on $\mathfrak{a},<_{J}$ is the partial order on $\prod \mathfrak{a}$ defined by

$$
f<_{J} g \Leftrightarrow\{\theta \in \mathfrak{a}: f(\theta)<g(\theta)\}=\mathfrak{a} \bmod J
$$

If $J=\{\emptyset\}$, then we write $<$.

2) $\operatorname{pcf}(\mathfrak{a})=\left\{\operatorname{tcf}\left(\prod \mathfrak{a},<_{J}\right): J\right.$ an ideal on $\mathfrak{a}$ and $\left(\prod \mathfrak{a},<_{J}\right)$ has true cofinality $\}$ (it is enough to consider maximal ideals).

3) $\operatorname{pcf}_{\theta \text {-complete }}(\mathfrak{a})=\left\{\operatorname{tcf}\left(\prod \mathfrak{a},<_{J}\right): J\right.$ is a $\theta$-complete ideal on $\mathfrak{a}$ and $\left(\prod \mathfrak{a},<_{J}\right)$ has true cofinality\}.

4) If $\mu$ is singular, i.e., $\mu>\operatorname{cf}(\mu)$, let $\operatorname{pp}(\mu)=\sup \left\{\operatorname{tcf}\left(\prod \mathfrak{a},<_{J}\right): \mathfrak{a} \subseteq \operatorname{Reg} \cap\right.$ $\mu,|\mathfrak{a}| \leq c f(\mu) \sup (\mathfrak{a})=\mu, J$ an ideal on $\mathfrak{a}$ such that $\lambda<\mu \Rightarrow \mathfrak{a} \cap \lambda \in J$ and $\left(\prod \mathfrak{a},<_{J}\right)$ has true cofinality $\}$.

5) $\operatorname{pp}_{\theta \text {-complete }}(\mu)$ is defined similarly restricting ourselves to $\theta$-complete ideals.

5.10 Thesis. [pp vs. power set] The power set function $\lambda \rightarrow 2^{\lambda}$ on regular cardinals is totally independent (the only rules are that it is not decreasing and $\left.\operatorname{cf}\left(2^{\lambda}\right)>\lambda\right)$. It is like hair colour today, easily manipulated, whereas $\operatorname{pp}(\lambda)$ and $\operatorname{pcf}(\mathfrak{a})$ are like the skeleton of set theory, not totally immune to "plastic surgery" (i.e., forcing starting with large cardinals) but at a great price (and pains).

So the chaotic behaviour of cardinal arithmetic comes from the static noise of the interference of two different phenomena:

One, the mapping $\lambda \rightarrow 2^{\lambda}$ for regular cardinals, which actually is very well understood: we know all the rules; anything fulfilling them is permissible. The second 
speaks about pcf theory: there are many mysteries that have not disappeared, but much is decided in ZFC (together they suffice; see 5.14). The claim that almost all is independent was wrong; the picture is more balanced.

So "the armies of God (fighting for resolution in ZFC)" and "the armies of the devil (trying to prove independence)" have advanced much and arrive at a new stand-off. So we should reformulate 5.5.

5.11 Dream. Find the laws of (infinite) cardinal exponentiation under pcf interpretation.

Note that $\operatorname{pcf}(\mathfrak{a})$ replaces the cardinality product $\prod \mathfrak{a}$ by a spectrum of possible cofinalities, a phenomena which has many honourable precedents (e.g., in decomposition into primes in algebraic extensions of $\mathbb{Z}$ ). On the calculus of pcf we know some rules:

5.12 Theorem. 1) $\operatorname{pcf}(\mathfrak{a})$ includes $\mathfrak{a}$ and has cardinality $\leq 2^{|\mathfrak{a}|}$ (and not merely $2^{2^{|\mathfrak{a}|}}$, which is the obvious upper bound being the number of ultrafilters on $\left.\mathfrak{a}\right)$.

2) $\operatorname{pcf}(\mathfrak{a})$ has a maximal member $\max \operatorname{pcf}(\mathfrak{a})$ which is equal to $\operatorname{cf}\left(\prod \mathfrak{a}\right)$.

3) If $\mathfrak{b} \subseteq \operatorname{pcf}(\mathfrak{a})$ and $|\mathfrak{b}|<\operatorname{Min}(\mathfrak{b})$, then $\operatorname{pcf}(\mathfrak{b}) \subseteq \operatorname{pcf}(\mathfrak{a})$.

In short, $\operatorname{pcf}(\mathfrak{a})$ is not as large as we may suspect; it has a last element which is a reasonable measure of $\prod \mathfrak{a}$. In fact it is $\operatorname{cf}\left(\prod \mathfrak{a},<_{J}\right)$ for $J$ the trivial ideal $\{\emptyset\}$. Moreover, pcf essentially acts like a closure operation; e.g., it is increasing.

Moreover

5.13 Theorem. 1) If a sequence $\left\langle\lambda_{i}: i<\aleph_{1}\right\rangle$ is increasing continuous with limit $\lambda$, then for some closed unbounded set $C \subseteq \aleph_{1}$ we have $\max \operatorname{pcf}\left\{\lambda_{i}^{+}: i \in C\right\}=\lambda^{+}$.

2) [Locality]: If $\mathfrak{b} \subseteq \operatorname{pcf}(\mathfrak{a}),|\mathfrak{b}|<\operatorname{Min}(\mathfrak{b}), \theta \in \operatorname{pcf}(\mathfrak{b})$, then for some $\mathfrak{c} \subseteq \mathfrak{b}$ of cardinality $\leq|\mathfrak{a}|$ we have $\theta \in \operatorname{pcf}(\mathfrak{c})$.

3) [No hole]: If $\mathfrak{a}$ is an interval of the class of regular cardinals (i.e., $\mathfrak{a}=\left\{\aleph_{\alpha+1}\right.$ : $\left.\alpha_{*} \leq \alpha<\beta\right\}$, so necessarily $\beta<\aleph_{\alpha_{*}}$ ), then $\operatorname{pcf}(\mathfrak{a})$ is an interval too (necessarily end-extending $\mathfrak{a}$; i.e., $\operatorname{pcf}(\mathfrak{a})$ is an initial segment of $\left.\left\{\aleph_{\alpha+1}: \alpha \geq \alpha_{*}\right\}\right)$.

What does this mean? The closure operation inside $\operatorname{pcf}(\mathfrak{a})$ has "character" at most the cardinality of $\mathfrak{a}$, and there are some forms of continuity and convexity.

5.14 Observation. $\lambda^{\kappa}=2^{\kappa}+\operatorname{cf}\left([\lambda]^{\kappa}, \subseteq\right)$.

In general

5.15 Thesis. The natural measures of $[\lambda]^{\kappa}$ can be expressed by cases of pp and of $2^{\lambda}$ for $\lambda$ regular.

The measures which come to my mind are $\lambda^{\kappa}, \operatorname{cf}\left([\lambda]^{\kappa}, \subseteq\right)$ from 5.7(2), and

$$
\begin{array}{r}
\lambda^{[\kappa]}=\operatorname{Min}\left\{|\mathcal{P}|: \mathcal{P} \subseteq[\lambda]^{\kappa} \text { and every subset of } \lambda \text { of cardinality } \kappa\right. \\
\text { is the union of }<\kappa \text { members of } \mathcal{P}\}, \\
\lambda^{<\kappa>}=\operatorname{Min}\left\{|\mathcal{P}|: \mathcal{P} \subseteq[\lambda]^{\kappa} \text { and every subset of } \lambda \text { of cardinality } \kappa\right. \\
\text { is included in the union of }<\kappa \text { members of } \mathcal{P}\}, \\
\lambda^{<\kappa>_{t r}}=\sup \left\{\left|\lim _{\kappa}(T)\right|: T \text { is a tree with } \leq \lambda \text { nodes and } \kappa \text { levels }\right\} .
\end{array}
$$

For example

5.16 Theorem. $\operatorname{cf}\left(\left[\aleph_{\omega}\right]^{\aleph_{0}}, \subseteq\right)=\operatorname{pp}\left(\aleph_{\omega}\right)=\max \operatorname{pcf}\left\{\aleph_{n}: n<\omega\right\}$. 
So pcf is a closure operation with some rules; those listed above were enough to prove a result, popular among my works: $\operatorname{pp}\left(\aleph_{\omega}\right)<\aleph_{\aleph_{4}}$; i.e. by 5.16 it is enough to investigate $\operatorname{pcf}\left\{\aleph_{n}: 0<n<\omega\right\}$ which, if the desired inequality $\left(\operatorname{pp}\left(\aleph_{\omega}\right) \geq \aleph_{\aleph_{4}}\right)$ fails, includes $\left\{\aleph_{\alpha+1}: \alpha<\aleph_{4}\right\}$.

Now pcf is a closure operation with several rules, but there is much we do not know. Where are the new lines between what is known and what we do not know? Locality may be a poor substitute to a positive answer to:

5.17 Question. Is $\operatorname{pcf}(\mathfrak{a})$ always of cardinality $\leq|\mathfrak{a}|$ ?

In the scale of the problems (in this direction) for which we do not know the answers, this question seems to me to lie in the middle.

Less hopeless for forcing are:

5.18 Question. Show the consistency of the failure of the Weak Hypothesis (WH), which means:

$(\mathrm{WH})_{1}$ for every cardinal $\lambda$ the following set is finite: $\{\mu<\lambda: \mu$ is singular of uncountable cofinality; that is $\aleph_{0}<\operatorname{cf}(\mu)<\mu$ and $\left.\operatorname{pp}_{\aleph_{1} \text {-complete }}(\mu) \geq \lambda\right\}$;

$(\mathrm{WH})_{2}$ for any cardinal $\lambda$ the following set is finite or at most countable: $\{\mu<\lambda: \operatorname{cf}(\mu)<\mu, \operatorname{pp}(\mu) \geq \lambda\}$.

5.19 Dream. Prove $(\mathrm{WH})$, i.e., $(\mathrm{WH})_{1}$ or $(\mathrm{WH})_{2}$.

This is really like a dream: I do not believe in it, but it is the best substitute for $\mathrm{GCH}$ which has not been proved impossible (essentially). The dual dream is not to prove its failure but essentially to prove that there are no more rules or at least to show that some pcf "bizarre" structures are possible:

5.20 The Forcer Dream. Prove the consistency of: There is a set a of regular cardinals $>|\mathfrak{a}|$ such that for some inaccessible (= regular limit uncountable) cardinal $\lambda$ we have $\lambda=\sup (\lambda \cap p c f(\mathfrak{a}))$. This will be enough for proving the consistent failure of $\operatorname{pcf}(\operatorname{pcf}(\mathfrak{a}))=\operatorname{pcf}(\mathfrak{a})$.

A quite reasonable hope is:

5.21 Question. For every $\lambda \geq \aleph_{\omega}$ there is $n<\omega$ for which we have:

for no $\mu<\lambda$ do we have $\operatorname{cf}(\mu)>\aleph_{n}$ and $\operatorname{pp}_{\operatorname{cf}(\mu) \text {-complete }}(\mu)>\lambda$.

A related statement for $\beth_{\omega}$ instead of $\aleph_{\omega}$ was proved in $[$ Sh 460 ] and put forward as a positive solution of Hilbert's first problem, i.e., $\mathrm{GCH}$; we have to see whether this is justified and/or accepted.

Note that 5.21 will be enough for deriving consequences of $2^{\aleph_{0}}>\aleph_{\omega}$, confirming the following.

5.22 Thesis. pcf theory will make failures of GCH semi-axioms; i.e., from some cardinal arithmetic equation or statement we shall prove many consequences. Note that whereas earlier $2^{\aleph_{0}}=\aleph_{1}$ and (in a few cases) $2^{\aleph_{0}}<\aleph_{\omega}$ has been used as an assumption, now there are some cases in which, e.g., $2^{\aleph_{0}}>\aleph_{\omega}$ is used.

In particular we may consider a singular strong limit cardinal $\mu$; any $\mu=\sum\left\{\mu_{n}\right.$ : $n \in \mathbb{N}\}$ with $2^{\mu_{n}}<\mu_{n+1}$ will do. We already know that $2^{\mu}=\mu^{+}$has serious consequences. Now we can hope that also $2^{\mu}>\mu^{+}$has. It implies that there is a $\mu^{+}$-free non-free Abelian group of cardinality $\mu^{+}$Sh:E12 5.5]. Also for some increasing sequence $\left\langle\lambda_{n}: n \in \mathbb{N}\right\rangle$ of regular cardinals with limit $\mu, \prod_{n} \lambda_{n}$ (ordered by $<_{J}$ for the ideal $J$ of bounded subsets of $\mathbb{N}$ ) has the true cofinality $\mu^{++}$(this is 
an instance of a major theme of Sh:g, that $\operatorname{pp}(\mu)$ is the "true" $\mu^{\mathrm{cf}(\mu)}$; see Sh:g, IX]). So it is not unreasonable to hope that this will provide a good dividing line.

The proofs in pcf depend very little on the advances in set theory from the sixties on (in Sh:g] it has been claimed that Cantor, arising from his grave, would be able to understand them; certainly he could understand the theorems). It may well be that the next stage in the evolution will be

5.23 Dream. Combine the methods of pcf and inner models to answer questions as above.

\section{$\S 6$ Concluding Remarks}

We discuss here, usually concisely and with scant references and background, various things. Not that they are less worthwhile than those discussed earlier; it's just that I have not found the time to write on them at leisure.

What is model theory? Classically you have a theory $T$ and the class $\operatorname{EC}(T)$ of all its models, i.e., structures of a fixed signature (e.g., for rings we have $+, \times, 0,1$ ) that satisfy $T$. The main case was $T$ first order; then the class is called first order or elementary, but other logics have been important as well. The point has been the interplay between what can be seen in $T$ (syntactical side) and what can be said on $\mathrm{EC}(T)$, semantical side. For example, a sentence $\psi$ (in first order logic) is preserved by submodels (a semantical property) iff $\psi$ is equivalent to a universal sentence (syntactical). See the introduction to the Berkeley Symposium in 1964.

There were some other frameworks. Some have suggested looking at less general structures. We may look at computable (= recursive) structures; we may look at Polish structures (i.e., for a separable complete metric space with the universal of the model and function continuous and relations, e.g., closed); we may look at Borel structures, at $\Sigma_{n}^{1}$-structures or structure from $\mathbf{L}[\mathbb{R}]$.

Could you choose any of them as the main framework? If you look at computable structures, are the atomic relations computable, or the first order definable ones computable? Why Borel and not closed (like Polish structures) or $F_{\sigma}$ ? Also dealing with Borel forces you to deal with $\Sigma_{1}^{1}$ and hence higher. $\mathbf{L}[\mathbb{R}]$ is a natural stopping point, but it is highly set-theoretic sensitive. We have a very different picture if $\mathbf{V}=\mathbf{L}$ on the one hand and if we assume $\mathrm{AD}_{\mathbf{L}[\mathbb{R}]}$ on the other hand (which requires high consistency strength). Hence

6.1 Model Theory Content Thesis. 1) While all those "restrictive frameworks" are interesting, their closure properties (or absence thereof) make them unnatural as the focus of model theory.

2) Also none of them is "the effective framework".

3) To advance with most of them we should better start with a general theory of non-elementary classes (see below).

4) Also if you are interested in universal first order classes or the existentially closed members of such classes, you better do it based on a theory for general first order classes.

6.2 ZFC vs. Model Theory Thesis. 1) It is preferable to have the theorem in $\mathrm{ZFC}$ or at least $\mathrm{ZFC}+$ a semi-axiom, like $\mathrm{CH}$.

2) If (1) fails, using a simple division of the $\lambda$ 's or proving one side in ZFC and the other by consistency is a reasonable substitute. 
We may strengthen first order logic. We may allow infinite conjunction, i.e. $\bigwedge_{n} \varphi_{n}$, while every formula still has finitely many free variables; this is $\mathbb{L}_{\aleph_{1}, \aleph_{0}}$ (similarly $\mathbb{L}_{\lambda^{+}, \aleph_{0}}$ if we allow $\bigwedge_{\alpha<\lambda} \varphi_{\alpha}$ ). Now $\mathbb{L}_{\aleph_{1}, \aleph_{0}}$ has many good properties; every sentence with an infinite model has a countable one (the D.L.S., downward Löwenheim-Skolem theorem), a completeness theorem (with an infinitary rule), and interpolation theorem (hence implicit definability implies explicit definability). $\mathbb{L}_{\lambda^{+}, \aleph_{0}}$ still has the D.L.S. but usually not the others.

For me a central topic of model theory is classification 3 theory for elementary classes, i.e., those defined as the class of models of a first order logic theory, the idea being to find natural dividing lines: positive theory for the lower halves (understanding the structure) and on the upper part (proving they have complicated models). Much has been done in the investigation of the theory of low classes (particularly stable but also simple and little on others). There is much to be done on them.

6.3 High Taxonomy Dream. Find a good dividing line (or lines) which is much higher than those mentioned above (see more in [Sh 702]).

6.4 The Mountain Air Thesis. The air on high mountains is clearer: many aspects are more transparent when we work in a more general thesis. In particular:

(a) Even if you are interested just in the model theory of specific structures (like the real and the p-adic fields), general model theory will help you.

(b) Even if you are interested just in countable models of first order classes $E C(T)$, you will be helped by looking at $\kappa$-saturated models of $T$ of cardinality $\geq \kappa$.

(c) It is good to prove that an "outside" property $\operatorname{Pr}_{T}(\lambda)$ which speaks of $\{M: M \models T$ has cardinality $\lambda\}$ of a first order $T$ does not depend on $\lambda$ by proving it equivalent to an "inside" syntactical property. It is also an excellent way to discover interesting syntactical properties of $T$ even if you have no interest in what occurs in every $\lambda$.

(d) Model theory of non-first-order classes will help the elementary case and may be more relevant than first order for investigating some natural uncountable structures.

6.5 The Specific Stability Dream. Find interesting natural first order classes $\mathrm{EC}(T)$ with an interesting model theory and in particular stability theory; I mean that the general methods of model theory are really combined with the investigation of those specific classes (not just quoting the results).

This has occurred for differentially closed fields (and to some extent, separably closed, not algebraically closed, fields).

6.6 Question. Are there infinite non-separably closed fields $F$ with stable $\operatorname{Th}(F)$ ?

\footnotetext{
${ }^{3}$ Note: It does not necessarily generalize stability theory. It is probably better to say taxonomy theory, as some people mistakenly interpret my intention in "classification theory" as finding the first order classes for which every model can be characterized up to isomorphism by (general) cardinal invariants (an important case, the major gap). It is harmless to let it also have this meaning.
} 
6.7 The Specific Taxonomy Dream. Carry out the classification program for the family of interesting specific classes $\operatorname{EC}(T)$, i.e., on $\left\{E C\left(T^{\prime}\right): T^{\prime} \supseteq T\right.$ is complete\}. This is not the same as 6.5; there we are interested in $T$ in which we can say much on $M \in E C(T)$. Here we look for dividing lines in the family of completions of $T$; e.g., $T=$ the theory of fields.

6.8 Problem. What are the right contexts for stability theory? Develop it in various specific cases; similarly for classification theory in general.

Various contexts we are considering:

(A) first order classes (with $\prec$ ),

$(B) \operatorname{EC}(T)$, for $T$ a universal first order theory with amalgamation under $\subseteq$,

$(C)$ the class of existentially closed models of a first order theory $T$ under $\subseteq$ (here we naturally do not have negation; i.e., the negation of a formula is an infinite disjunction of ones),

(D) $D$-homogeneous models with $\prec$ (so we restrict $\operatorname{EC}(T)$ to $\{M:\{\operatorname{tp}(\bar{a}, \emptyset, M)$ : $\left.\left.\bar{a} \in{ }^{\omega>} M\right\} \subseteq D\right\}$ and assume a strong form of amalgamation),

(E) universal classes [Sh 300],

(F) $\operatorname{EC}(T), T \subseteq \mathbb{L}_{\lambda^{+}, \omega}$ and $\prec \Delta, \Delta$ a fragment of $\mathbb{L}_{\lambda^{+}, \omega}$,

$(G)$ abstract elementary class with amalgamation,

$(H)$ abstract elementary class with no maximal model,

(I) abstract elementary class,

$(J)$ good frames and relatives Sh 705].

Note that one of the desired properties is having good closure properties (e.g., for interpreting groups), so a wider context may be of interest, as it has better closure properties.

A hard test is the following.

6.9 The Main Gap Question. Prove a form of the main gap for $\psi \in \mathbb{L}_{\lambda^{+}, \omega}$ (or just $\mathbb{L}_{\aleph_{1}, \aleph_{0}}$ or generally an abstract elementary class); i.e., for every such $\psi$ either $I(\lambda, \psi)>\lambda$ (see below) for every $\lambda$ large enough or there is an ordinal $\gamma$ such that for every ordinal $\alpha, I\left(\aleph_{\alpha}, \psi\right) \leq \beth_{\gamma}(|\alpha|)$.

6.10 Definition. $I(\lambda, \psi)=\{M / \cong: M \models \psi\}$; similarly $I(\lambda, K)$ for $K$ a class of models.

As in the case of the first order, the intention is

6.11 Main Gap a Good Test Thesis. Solving 6.9 will force you to develop a theory and find interesting definitions and theorems for it.

In the seventies soft model theory (i.e., with the logic as a variable) was very popular (see $[\mathrm{BF}]$ ) but has since gone out of fashion, probably as it seemed that there were many counterexamples and few theorems. I do not see this as a final verdict.

6.12 Dream. Find natural properties of logics and nontrivial implications between them (giving a substantial mathematical theory, of course).

6.13 Dream. Find a new logic with good model theory (like compactness, completeness theorem, interpolation and those from 6.12) and strong expressive power, preferably concerning other parts of mathematics; possibly specifically derive for them (see [Sh 702]. 
6.14 Problem. Develop the model theory of first order classes $\mathrm{EC}(T)$ with $T$ having the finite model property (i.e., every finite subset of $T$ has a finite model).

6.15 Problem. Develop and investigate a logic for "polynomial invariants for graphs and general structures" (there are many worthwhile definitions of polynomials (invariants) for a graph, which depend on the isomorphism type only).

In fact, I am not fond of set theory without choice, nevertheless:

6.16 Problem. Develop combinatorial set theory for universes with a limited amount of choice (see [Sh 666] $)$.

6.17 Problem. Develop descriptive set theory for ${ }^{\kappa} \mu$, in particular ${ }^{\omega} \mu, \mu$ strong limit singular of cofinality $\aleph_{0}$ (see discussion in Sh 724]; probably we first of all need good questions, as a straight generalization of properties may not succeed).

6.18 Dream. Try to formalize and really say something on mathematical beauty and depth. Of course (length of proof)/(length of theorem) is in the right direction, etc.

6.19 Dream. Make a reasonable mathematical theory when we restrict ourselves to the natural numbers up to $n$, where $n$ is a specific natural number (say $2^{2^{100}}+1$ ) (e.g., thinking our universe is discrete with this size).

\section{REFERENCES}

[Baxx] Tomek Bartoszyński. Invariants of Measure and Category. In M. Foreman, A. Kanamori, and M. Magidor, editors, Handbook of Set Theory. Kluwer, to appear.

$[\mathrm{BF}] \quad J o n$ Barwise and Solomon Feferman (editors). Model-theoretic logics. Springer Verlag, Heidelberg-New York, 1985. MR 87g:03033

[Bsxx] Andreas Blass. Combinatorial Cardinal Characteristics of the Continuum. In M. Foreman, A. Kanamori, and M. Magidor, editors, Handbook of Set Theory. Kluwer, to appear.

[DeJ] Keith J. Devlin and Ronald B. Jensen. Marginalia to a theorem of Silver. In G. H. Müller, A. Oberschelp, and K. Potthoff, editors, Proceedings of the Logic Colloquium Kiel 1974, volume 499 of Lecture Notes in Mathematicas, pages 115-142, Berlin, 1975. Springer. MR 58:235]

[DJ1] A. Dodd and Ronald B. Jensen. The Core model. Annals of Math Logic, 20:43-75, 1981. MR 82i:03063

[DjSh:659] M. Džamonja and S. Shelah. Universal graphs at the successor of singular cardinal. J. of Symbolic Logic, to appear in 2003.

[EM] Paul C. Eklof and Alan Mekler. Almost free modules: Set theoretic methods, volume 46 of North Holland Mathematical Library. North-Holland Publishing Co., Amsterdam, 1990. MR 92e:20001

[EM02] Paul C. Eklof and Alan Mekler. Almost free modules: Set theoretic methods, volume 65 of North Holland Mathematical Library. North-Holland Publishing Co., Amsterdam, 2002. Revised Edition.

[EHR] Paul Erdős, Andras Hajnal, and Richard Rado. Partition relations for cardinal numbers. Acta Math. Acad. Sci. Hung., 16:93-196, 1965. MR 34:2475

[GH] Fred Galvin and Andras Hajnal. Inequalities for cardinal powers. Annals Math., 101:491-498, 1975. MR 51:12535

[GiSh 582] Moti Gitik and Saharon Shelah. More on real-valued measurable cardinals and forcing with ideals. Israel Journal of Mathematics, 124:221-242, 2001. math.LO/9507208. MR 2002g:03110

\footnotetext{
${ }^{4}$ Here, as elsewhere, e.g., $6.19,4.1$, the dream is not to suggest a reasonable framework for this, but to build a mathematical theory.
} 
[GoSh 448] Martin Goldstern and Saharon Shelah. Many simple cardinal invariants. Archive for Mathematical Logic, 32:203-221, 1993. math.LO/9205208. MR 94c:03064

[J] Thomas Jech. Set theory. Academic Press, New York, 1978. MR 80a:03062

[KS81] Jussi Ketonen and Robert Solovay. Rapidly growing Ramsey functions. Ann. Math., II. Ser., 113:267-314, 1981. MR 84c:03100

[KP82] Laurie Kirby and Jeff Paris. Accessible independence results for Peano arithmetic. Bull. Lond. Math. Soc., 14:285-293, 1982. MR 83j:03096

[Mg2] Menachem Magidor. Chang's conjecture and powers of singular cardinals. J. Symbolic Logic, 42:272-276, 1977. MR 58:5221

[Mg1] Menachem Magidor. On the singular cardinals problem II. Annals Math., 106:517$547,1977$.

[FOM] Stephen G. Simpson (moderator). E-mail list for discussing Foundations of Mathematics. http://www. math.psu.edu/simpson/fom/.

[PH77] Jeff Paris and Leo Harrington. A mathematical incompleteness in Peano Arithmetic. In Jon Barwise, editor, Handbook of Mathematical Logic, pages 1133-1142. NorthHolland Publishing Co., Amsterdam-New York-Oxford, 1977. MR 56:15351

[Sh:E12] Saharon Shelah. Analytical Guide and Corrections to Sh:g]. math.LO/9906022.

[Sh:E16] Saharon Shelah. The future of set theory. In Haim Judah, editor, Set Theory of the Reals, volume 6 of Israel Mathematical Conference Proceedings. Proceedings of the Winter Institute held at Bar-Ilan University, Ramat Gan, January 1991. MR 94i:03002

[Sh 413] Saharon Shelah. More Jonsson Algebras. Archive for Mathematical Logic, accepted. math.LO/9809199.

[Sh 724] Saharon Shelah. On nice equivalence relations on ${ }^{\lambda}$ 2. Archive for Mathematical Logic, accepted. math.LO/0009064.

[Sh 702] Saharon Shelah. On what I do not understand (and have something to say), model theory. Mathematica Japonica, 51:329-377, 2002. math.LO/9910158.

[Sh 771] Saharon Shelah. Polish Algebras shy from freedom. Israel Journal of Mathematics, submitted.

[Sh 705] Saharon Shelah. Toward classification theory of good $\lambda$ frames and abstract elementary classes, preprint.

[Sh:b] Saharon Shelah. Proper forcing, volume 940 of Lecture Notes in Mathematics. Springer-Verlag, Berlin-New York, xxix+496 pp, 1982. MR 84h:03002

[Sh 170] Saharon Shelah. On logical sentences in PA. In Logic colloquium '82 (Florence, 1982), volume 112 of Stud. Logic Found. Math, pages 145-160. North-Holland, AmsterdamNew York, 1984. MR 86g:03097

[Sh 111] Saharon Shelah. On power of singular cardinals. Notre Dame Journal of Formal Logic, 27:263-299, 1986. MR 87j:04006

[Sh 300] Saharon Shelah. Universal classes. In Classification theory (Chicago, IL, 1985), volume 1292 of Lecture Notes in Mathematics, pages 264-418. Springer, Berlin, 1987. Proceedings of the USA-Israel Conference on Classification Theory, Chicago, December 1985; ed. Baldwin, J.T. MR 91k:03088

[Sh:g] Saharon Shelah. Cardinal Arithmetic, volume 29 of Oxford Logic Guides. Oxford University Press, 1994. MR 96e:03001

[Sh:f] Saharon Shelah. Proper and improper forcing. Perspectives in Mathematical Logic. Springer, 1998. MR 98m:03002

[Sh 666] Saharon Shelah. On what I do not understand (and have something to say). Fundamenta Mathematicae, 166:1-82, 2000. math.LO/9906113.

[Sh 460] Saharon Shelah. The Generalized Continuum Hypothesis revisited. Israel Journal of Mathematics, 116:285-321, 2000. math.LO/9809200. MR 2001g:03095

[ShSt 419] Saharon Shelah and Lee Stanley. Filters, Cohen Sets and Consistent Extensions of the Erdős-Dushnik-Miller Theorem. Journal of Symbolic Logic, 65:259-271, 2000. math.LO/9709228. MR 2001j:03085

[Sh:E25] Shelah, Saharon. You Can Enter Cantor's Paradise! In Proceedings of the Erdös Conference. Budapest'99. math.LO/0102056.

[Si] Jack Silver. On the singular cardinal problem. In Proceedings of the International Congress of Mathematicians, volume I, pages 265-268, Vancouver, 1974. MR 55:2576 
[Wd00] Hugh Woodin. The Continuum Hypothesis, Part I. Notices of the American Mathematical Society, 48:567-576, 2001.

[Woxx] Hugh Woodin. The Axiom of Determinacy, Forcing Axioms and the Nonstationary Ideal, volume 1 of de Gruyter Series in Logic and its Applications. De Gruyter, in press.

Institute of Mathematics, The Hebrew University, Jerusalem, Israel

E-mail address: shelah@math.huji.ac.il

Mathematics Department, Rutgers University-New Brunswick, Piscataway, New JerSEY $08854-8019$ 